TRANSACTIONS OF THE

AMERICAN MATHEMATICAL SOCIETY

Volume 354, Number 12, Pages 5027-5038

S 0002-9947(02)03113-6

Article electronically published on August 1, 2002

\title{
LOCATION OF THE FERMAT-TORRICELLI MEDIANS OF THREE POINTS
}

\author{
CARLOS BENÍTEZ, MANUEL FERNÁNDEZ, AND MARÍA L. SORIANO
}

\begin{abstract}
We prove that a real normed space $X$ with $\operatorname{dim} X \geq 3$ is an inner product space if and only if, for every three points $u, v, w \in X$, the set of points at which the function $x \in X \rightarrow\|u-x\|+\|v-x\|+\|w-x\|$ attains its minimum (called the set of Fermat-Torricelli medians of the three points) intersects the convex hull of these three points.
\end{abstract}

\section{INTRODUCTION}

The classical Fermat problem [11 p. 153] for three points $u, v, w$ of the Euclidean plane $X$ deals with a fourth point such that the sum of its distances to the three given points is a minimum, i.e., a point $z \in X$ such that

$$
\|u-z\|+\|v-z\|+\|w-z\| \leq\|u-x\|+\|v-x\|+\|w-x\| \text {, for every } x \in X \text {. }
$$

The point $z$, called the Fermat-Torricelli median of the three points, is unique and belongs to their convex hull, $\operatorname{co}(u, v, w)$. Moreover, a historical theorem of Torricelli and Cavalieri says that either $z$ is one of the three points, or pairwise the segments $[z, u],[z, v]$, and $[z, w]$ form angles of $2 \pi / 3$ (see, e.g., [14]).

Suppose now that we are not in the Euclidean plane, but in a real normed space $X$ and that $Z^{1}(u, v, w)$ denotes the set of Fermat-Torricelli medians of the three points $u, v, w \in X$. It is easy to verify that if $X$ is an inner product space, then $Z^{1}(u, v, w)$ has, as in the classical problem, a unique point that belongs to $\operatorname{co}(u, v, w)$. In the general case, it is also easy to see that $Z^{1}(u, v, w)$ is closed and convex, but there arise, amongst others (effective calculation, ... ), three basic problems:

Existence. It is possible that $Z^{1}(u, v, w)=\emptyset$. An example due to Baronti, Casini and Papini [2] is the space

$$
X=\left\{x \in c_{0}: \sum_{n=1}^{4} x_{n}+\sum_{n=5}^{\infty} \frac{1}{2^{n-4}} x_{n}=0\right\},
$$

endowed with the sup norm and $u, v, w$ the points $(-1,0,0,1,0, \ldots),\left(0,-1,0, \frac{1}{2}, 1\right.$, $0, \ldots),\left(0,0,-1, \frac{1}{2}, 1,0, \ldots\right)$. (In fact, Veselý [19] proved that either $X$ is reflexive or there exist $u, v, w \in X$ and an equivalent norm in $X$ such that $Z^{1}(u, v, w)=\emptyset$.)

Received by the editors November 27, 2000 and, in revised form, May 17, 2001.

2000 Mathematics Subject Classification. Primary 46B20, 46C15, $90 \mathrm{~B} 85$.

Key words and phrases. Optimal location, medians, inner product spaces.

Partially supported by MCYT (Spain) and FEDER, BFM2001-0849. 
Uniqueness. The set $Z^{1}(u, v, w)$ may have more than one element. A simple example, for $X$ the space $\mathbb{R}^{2}$ endowed with the norm

$$
\left\|\left(x_{1}, x_{2}\right)\right\|= \begin{cases}\sup \left(\left|x_{1}\right|,\left|x_{2}\right|\right), & \text { if } x_{1} x_{2} \geq 0 \\ \left|x_{1}\right|+\left|x_{2}\right|, & \text { if } x_{1} x_{2}<0\end{cases}
$$

is $Z^{1}((1,0),(-1,0),(1,2))=\operatorname{co}((1,0),(-1,0),(1,2))$.

Location. It may be that

$$
Z^{1}(u, v, w) \neq \emptyset, \quad Z^{1}(u, v, w) \cap \operatorname{co}(u, v, w)=\emptyset .
$$

For instance, when $X=\ell_{1}^{3}, Z^{1}((1,0,0),(0,1,0),(0,0,1))=\{(0,0,0)\}$. (It is known 20 that if $Z^{1}(u, v, w) \cap \operatorname{aff}(u, v, w) \neq \emptyset$, then $Z^{1}(u, v, w) \cap \operatorname{co}(u, v, w) \neq \emptyset$. Therefore, since $Z^{1}(u, v, w) \neq \emptyset$ when $X$ is finite-dimensional, it is not possible that $Z^{1}(u, v, w) \cap \operatorname{co}(u, v, w)=\emptyset$ when $\operatorname{dim} X<3$.)

In this paper we give an affirmative answer to a plausible conjecture relative to location. Namely, we shall prove that if a real normed space $X$ of dimension $\geq 3$ satisfies

$$
Z^{1}(u, v, w) \cap \operatorname{co}(u, v, w) \neq \emptyset, \quad \text { for every } u, v, w \in X,
$$

then it is an inner product space.

We denote the above hypothesis GK1 in honour of Garkavi and Klee who, forty years ago [12, 13], [1, p. 119], obtained an analogous result for the hypothesis

$$
Z^{\infty}(u, v, w) \cap \operatorname{co}(u, v, w) \neq \emptyset, \quad \text { for every } u, v, w \in X,
$$

where $Z^{\infty}(u, v, w)$ is the set of Chebyshev centers of $u, v, w$, i.e., the set of points $z \in X$ such that, for every $x \in X$,

$$
\sup (\|u-z\|,\|v-z\|,\|w-z\|) \leq \sup (\|u-x\|,\|v-x\|,\|w-x\|) .
$$

Also, a characteristic property of real inner product spaces of dimension $\geq 3$ [3, 4] is the hypothesis GKp, with $p>1$, relative to the set $Z^{p}(u, v, w)$ of points $z \in X$ such that, for every $x \in X$,

$$
\|u-z\|^{p}+\|v-z\|^{p}+\|w-z\|^{p} \leq\|u-x\|^{p}+\|v-x\|^{p}+\|w-x\|^{p} .
$$

Actually it is not difficult to reduce the hypothesis GKp, with $1<p \leq+\infty$, to the classical Brunn-Blaschke-Kakutani theorem, which states that a real normed space of dimension $\geq 3$ is an inner product space if and only if there are norm-1 linear projections of $X$ onto any of its 2-dimensional subspaces (see, e.g., [1, p. 99]).

However, there seems to be no straightforward analogous reduction for the case GK1, i.e., relative to the oldest kind of center, which is the most interesting in a variety of fields (operations research, economics,...).

Some partial answers on the subject of this paper have been obtained by Durier [8], who proved that a characteristic of real inner product spaces of dimension $\geq 3$ is the hypothesis

$$
Z_{\alpha_{1}, \alpha_{2}, \alpha_{3}, \alpha_{4}}^{1}\left(u_{1}, u_{2}, u_{3}, u_{4}\right) \cap \operatorname{co}\left(u_{1}, u_{2}, u_{3}, u_{4}\right) \neq \emptyset,
$$

for every four nonnegative weights $\alpha_{1}, \alpha_{2}, \alpha_{3}, \alpha_{4}$ and every four points $u_{1}, u_{2}, u_{3}, u_{4}$ $\in X$, where $z \in Z_{\alpha_{1}, \alpha_{2}, \alpha_{3}, \alpha_{4}}^{1}\left(u_{1}, u_{2}, u_{3}, u_{4}\right)$ means that

$$
\sum_{i=1}^{4} \alpha_{i}\left\|u_{i}-z\right\| \leq \sum_{i=1}^{4} \alpha_{i}\left\|u_{i}-x\right\|, \quad \text { for every } x \in X,
$$


and Lewicki [16, who proved the same for three, instead of four, weights and points. But whether GK1 characterizes real inner product spaces of dimension $\geq 3$ is expressly mentioned as an open question in [9], [20], 7, p. 98], and [6, p. 274], among others.

\section{Preliminary Results}

In this section we take $X$ to be any real normed space of dimension $\geq 3$.

Let $S$ and $S^{*}$ be the unit spheres of $X$ and its topological dual $X^{*}$, respectively. For $u \in X$ and $f \in X^{*}$, write $J u=\left\{f \in S^{*}: f(u)=\|u\|\right\}$ and $J^{*} f=\{u \in S$ : $f(u)=\|f\|\}$. It is well-known that:

(i) For $u \neq 0, J u$ is a nonempty, closed and convex subset of $S^{*}$, and, for $f \neq 0$, $J^{*} f$ is a closed and convex subset of $S$, which is nonempty (for every $f \neq 0$ ) when $X$ is reflexive.

(ii) $X$ is smooth if and only if $J u$ has a unique element for every $u \neq 0$ or, equivalently, $J^{*} f \cap J^{*} g=\emptyset$ when $f$ and $g$ are linearly independent.

(iii) $X$ is rotund if and only if $J u \cap J v=\emptyset$ when $u$ and $v$ are linearly independent or, equivalently, $J^{*} f$ has at most one element for every $f \neq 0$.

(In the case of uniqueness we shall write $J u=f$ or $J^{*} f=u$.)

Let $u, v, w \in X$. It is obvious that $z \in Z^{1}(u, v, w)$ if and only if

$$
(z, z, z) \in P_{\operatorname{diag} X^{3}}^{1}(u, v, w),
$$

i.e., $(z, z, z)$ is a best approximation to $(u, v, w)$ from the linear subspace $\operatorname{diag} X^{3}=$ $\{(x, x, x): x \in X\}$, when $X^{3}$ is endowed with the norm $\|(u, v, w)\|_{1}=\|u\|+\|v\|+$ $\|w\|$.

According to [15] p. 8] the following duality result was first discovered by Vecten in 1811 and Fasbender [10] in 1846 for the classical Fermat-Torricelli problem. Since then, it has been frequently rediscovered in different contexts.

Proposition 1. $0 \in Z^{1}(u, v, w)$ if and only if there exist $f \in J u, g \in J v, h \in J w$ such that $f+g+h=0$.

Proof. It easily follows from the Hahn-Banach theorem (see, e.g., [18, p. 18]) that

$$
(0,0,0) \in P_{\operatorname{diag} X^{3}}^{1}(u, v, w)
$$

is equivalent to the existence of $(f, g, h) \in J(u, v, w)$, which is null on $\operatorname{diag} X^{3}$. That is,

$$
\begin{aligned}
\|(f, g, h)\| & =\sup (\|f\|,\|g\|,\|h\|)=1, \\
(f, g, h)(u, v, w) & =f(u)+g(v)+h(w)=\|u\|+\|v\|+\|w\|, \\
(f, g, h)(x, x, x) & =f(x)+g(x)+h(x)=0, \quad \text { for every } x \in X .
\end{aligned}
$$

Then the inequality $f(u)+g(v)+h(w) \leq\|f\|\|u\|+\|g\|\|v\|+\|h\|\|w\|$ shows that the first and second equalities above are equivalent to $f \in J u, g \in J v, h \in J w$.

Remark 2. Analogous arguments show that $0 \in Z^{p}(u, v, w)$, with $1<p<+\infty$, if and only if there are $f \in J u, g \in J v, h \in J w$ such that $\|u\|^{p-1} f+\|v\|^{p-1} g+$ $\|w\|^{p-1} h=0$. Then, the next corollary is not true for $p>1$ and, curiously, this is the reason for the greater difficulty of the proof that GKp implies i.p.s. for the case $p=1$.

Corollary 3. If $0 \in Z^{1}(u, v, w)$ and $\lambda, \mu, \nu$ are nonnegative real numbers, then $0 \in Z^{1}(\lambda u, \mu v, \nu w)$. 
Corollary 4. $z \in Z^{1}(u, v, w)$ if and only if there exist $f \in J(u-z), g \in J(v-z)$, $h \in J(w-z)$ such that $f+g+h=0$.

Proof. $z \in Z^{1}(u, v, w)$ is equivalent to $0 \in Z^{1}(u-z, v-z, w-z)$.

Corollary 5. Let $f, g, h \in S^{*}$ be such that $f+g+h=0$, let $u \in J^{*} f, v \in J^{*} g, w \in$ $J^{*} h,\left(\right.$ i.e., $\left.0 \in Z^{1}(u, v, w)\right)$, and let $z$ be distinct from $u, v, w$. Then $z \in Z^{1}(u, v, w)$ if and only if

$$
\frac{u-z}{\|u-z\|} \in J^{*} f, \quad \frac{v-z}{\|v-z\|} \in J^{*} g, \quad \frac{w-z}{\|w-z\|} \in J^{*} h .
$$

Lemma 6. Let $P$ be a 2-dimensional subspace of $X^{*}$ and $f \in S^{*} \cap P$. Then:

(i) There exist $g, h \in S^{*} \cap P$ such that $f+g+h=0$.

(ii) If $f$ is non-parallel to a segment of $S^{*} \cap P$ of length $>1$, then the $g$ and $h$ above are unique.

(iii) If $f$ is non-parallel to a segment of $S^{*} \cap P$ of length $\geq 1$, and $g$ and $h$ are as above, then any $v \in J^{*} g$ and $w \in J^{*} h$ are linearly independent.

Proof. (i) A simple continuity argument shows the existence of $g \in S^{*} \cap P$ such that $\|f+g\|=1$.

(ii) If $g, h, g^{\prime}, h^{\prime} \in S^{*} \cap P$ are such that $f+g+h=f+g^{\prime}+h^{\prime}=0$, with $g \neq g^{\prime}$ and both in the same half plane of $P$ relative to $f$, then the segments parallel to $f$, $\left[g,-h^{\prime}\right]$ and $\left[g^{\prime},-h\right]$, lie on $S^{*} \cap P$ and either $\left\|g+h^{\prime}\right\|>1$ or $\left\|g^{\prime}+h\right\|>1$.

(iii) If $v \in J^{*} g$ and $-v \in J^{*} h$, then $[g,-h] \subset S^{*}, g+h=-f$ and $\|g+h\|=1$.

Lemma 7. If $f, g, h \in S^{*}$ are such that $f+g+h=0$ and $u \in J^{*} f, v \in J^{*} g$, $w \in J^{*} h$ are such that $\operatorname{dim}[\operatorname{span}(u, v, w)]=2$, then $0 \in \operatorname{co}(u, v, w)$.

Proof. Let $r, s, t \in \mathbb{R}$ be such that $r+s+t=1$ and $r u+s v+t w=0$. Since $g(v)=h(w)=1$ and $g(u), g(w), h(u), h(v) \in[-1,0]$, it easily follows from

$$
\begin{aligned}
r+s+t & =1, \\
r g(u)+s g(v)+t g(w) & =0, \\
r h(u)+s h(v)+t h(w) & =0
\end{aligned}
$$

that $r, s, t$ are nonnegative.

\section{Main Results}

From now on we shall suppose that $X$ satisfies GK1 and, as a consequence of the next lemma, that $\operatorname{dim} X=3$.

Lemma 8. A real normed space $X$ of dimension $\geq 3$ satisfies $G K 1$ if and only if all of its 3-dimensional subspaces satisfy GK1.

Proof. The necessity is obvious. Conversely, suppose that $X$ does not satisfy GK1, i.e., that there are $u, v, w \in X$ such that $Z^{1}(u, v, w) \cap \operatorname{co}(u, v, w)=\emptyset$. Then $Z^{1}(u-w, v-w, 0) \cap \operatorname{co}(u-w, v-w, 0)=\emptyset$ and, since $\operatorname{co}(u-w, v-w, 0)$ is compact, there is $y \notin \operatorname{co}(u-w, v-w, 0)$ such that, for every $x \in \operatorname{co}(u-w, v-w, 0)$,

$$
\|u-w-y\|+\|v-w-y\|+\|y\|<\|u-w-x\|+\|v-w-x\|+\|x\| .
$$

That is,

$$
Z_{M}^{1}(u-w, v-w, 0) \cap \operatorname{co}(u-w, v-w, 0)=\emptyset,
$$


where $M=\operatorname{span}(u-w, v-w, y)$ and $Z_{M}^{1}(u-w, v-w, 0)$ denotes the set of points $z \in M$ such that

$$
\|u-z\|+\|v-z\|+\|w-z\| \leq\|u-x\|+\|v-x\|+\|w-x\|,
$$

for every $x \in M$.

As a consequence of this lemma and of the well-known fact that $X$ is an inner product space if and only if so are all of its 3-dimensional subspaces, to prove that GK1 characterizes real inner product spaces of dimension $\geq 3$, it suffices to consider the case in which $\operatorname{dim} X=3$.

With this convention $(\operatorname{dim} X=3)$ we can freely use the nice properties that $S$ and $S^{*}$ are compact, $Z^{1}(u, v, w) \neq \emptyset$ for every $u, v, w \in X$, and $J^{*} f \neq \emptyset$ for every $f \in X^{*}$.

Although the next proposition has a boring repetitive proof, it is one of the cornerstones of our argument. It will be easy to see that the last three of the four different cases that we shall consider in its proof correspond to highly non-regular spheres in $S^{*}$ that contain some segment of length $\geq 1$.

Proposition 9. Suppose that $X$ satisfies $G K 1$ and $f, g, h \in S^{*}$ are such that $f+$ $g+h=0$. Then, for any $u \in J^{*} f$ and $v \in J^{*} g$, there exists $w \in J^{*} h$ such that $\operatorname{dim}[\operatorname{span}(u, v, w)]=2$.

Proof. It is obvious when $u=-v$. (This is the case, for example, when $X=\ell_{\infty}^{3}$ and $f=(1 / 2,1 / 2,0), g=(-1,0,0), h=(1 / 2,-1 / 2,0), u=(1,1,0), v=(-1,-1,0)$.

In general, i.e., when $\operatorname{dim}[\operatorname{span}(u, v)]=2$, let $p \in S^{*}$ be such that $\operatorname{ker} p=$ $\operatorname{span}(u, v)$. In three of the four cases that follow we shall prove that there is a $w \in J^{*} h \cap \operatorname{ker} p$.

Suppose, on the contrary, that $p(x) \neq 0$ for every $x \in J^{*} h$. Since $J^{*} h$ is convex, the sign of $p$ may be chosen so that $\left\{p(x): x \in J^{*} h\right\} \subset \mathbb{R}_{+}$and, since $J^{*} h$ is a non-void and compact set, there is a $\bar{w} \in J^{*} h$ such that

$$
p(\bar{w})=\inf \left\{p(x): x \in J^{*} h\right\}>0 .
$$

An immediate consequence of $f+g+h=0$ is that $h(u) \leq 0$ and $h(v) \leq 0$, and we shall consider four possible cases with different (but similar) lines of argument.

Case $1(h(u)<0$ and $h(v)<0)$. It follows from Proposition 1 and Corollary 3 that $0 \in Z^{1}(u, v, 2 \bar{w})$. But $0 \notin \operatorname{co}(u, v, 2 \bar{w})$, and it follows from

$$
\begin{aligned}
\|u-2 \bar{w}\| & +\|v-2 \bar{w}\| \geq h(2 \bar{w}-u)+h(2 \bar{w}-v) \\
& =4-h(u)-h(v)>4=\|u\|+\|v\|+\|2 \bar{w}\|
\end{aligned}
$$

that $2 \bar{w} \notin Z^{1}(u, v, 2 \bar{w})$.

Then, by GK1, there exist $r \geq 0, s \geq 0,1>t \geq 0$ such that $r+s+t=1$ and

$$
z=r u+s v+t 2 \bar{w} \in Z^{1}(u, v, 2 \bar{w}) .
$$

Therefore, by Corollary $5, \frac{2 \bar{w}-z}{\|2 \bar{w}-z\|} \in J^{*} h$. But

$$
p\left(\frac{2 \bar{w}-z}{\|2 \bar{w}-z\|}\right)=\frac{2(1-t) p(\bar{w})}{2(1-t)-r h(u)-\operatorname{sh}(v)}<p(\bar{w}),
$$

in contradiction with the definition of $\bar{w}$.

(Note that in the next three cases the common hypothesis $h(u)=0$ implies that $(f-g)(u)=2$ and, hence, that the segment $[f,-g]$ of length 1 lies on $S^{*}$.) 
Case $2(h(u)=0, h(v)<0$ and $f(\bar{w})<0)$. As in Case $1,0 \in Z^{1}(u, \lambda v, \bar{w})$ for every $\lambda>0$, but $0 \notin \operatorname{co}(u, \lambda v, \bar{w})$.

For $y=r u+(1-r) \bar{w}$, with $0 \leq r \leq 1$, and $\lambda>0$ such that $\lambda f(v)-f(\bar{w})>0$, we have that

$$
\begin{aligned}
\|u-y\| & +\|\lambda v-y\|+\|\bar{w}-y\|=\|u-\bar{w}\|+\|\lambda v-y\| \\
& \geq f(u-\bar{w})+\sup (g(\lambda v-y), h(y-\lambda v)) \\
& =1-f(\bar{w})+\sup (1+\lambda+(1-r) f(\bar{w}), 1-r-\lambda h(v)) \\
& =\sup (2+\lambda-r f(\bar{w}), 2+\lambda-r-f(\bar{w})+\lambda f(v)) \\
& >2+\lambda=\|u\|+\|\lambda v\|+\|\bar{w}\| .
\end{aligned}
$$

In other words, $y \notin Z^{1}(u, \lambda v, \bar{w})$ and, hence, there exist $r \geq 0, s>0$, and $t \geq 0$ such that $r+s+t=1$ and

$$
z=r u+s \lambda v+t \bar{w} \in Z^{1}(u, \lambda v, \bar{w}),
$$

from which follows the contradiction that $\frac{\bar{w}-z}{\|\bar{w}-z\|} \in J^{*} h$ and

$$
p\left(\frac{\bar{w}-z}{\|\bar{w}-z\|}\right)=\frac{(1-t) p(\bar{w})}{1-t-s \lambda h(v)}<p(\bar{w}) .
$$

Case $3(h(u)=0, h(v)<0$, and $f(\bar{w})=0)$. If $\|\bar{w}-u\|>1$, then, for every $y=$ $r u+(1-r) \bar{w}$ with $0 \leq r \leq 1$, we have that

$$
\begin{gathered}
\|u-y\|+\|v-y\|+\|\bar{w}-y\|=\|\bar{w}-u\|+\|v-y\| \\
>1+g(v-y)=3=\|u\|+\|v\|+\|\bar{w}\|,
\end{gathered}
$$

i.e., $y \notin Z^{1}(u, v, \bar{w})$, and the proof follows as in Case 2 .

If $\|\bar{w}-u\|=1$, then $\bar{w}-u \in J^{*} h$ and $0 \in Z^{1}(u, v, \bar{w}-u)$, but $0 \notin \operatorname{co}(u, v, \bar{w}-u)$.

For $0 \leq r \leq 1$ and $y=r u+(1-r)(\bar{w}-u)$ the inequalities

$$
\begin{aligned}
\|u-y\| & +\|v-y\|+\|\bar{w}-u-y\|=\|\bar{w}-2 u\|+\|v-y\| \\
& \geq f(2 u-\bar{w})+\sup (g(v-y), h(y-v)) \\
& =2+\sup (1+r, 1-r-h(v))>3=\|u\|+\|v\|+\|\bar{w}-u\|
\end{aligned}
$$

show that $y \notin Z^{1}(u, v, \bar{w}-u)$. Then, there exist $r \geq 0, s>0, t \geq 0$ such that

$$
z=r u+s v+t(\bar{w}-u) \in Z^{1}(u, v, \bar{w}-u)
$$

and, hence, $\frac{\bar{w}-u-z}{\|\bar{w}-u-z\|} \in J^{*} h$, which is in contradiction with

$$
p\left(\frac{\bar{w}-u-z}{\|\bar{w}-u-z\|}\right)=\frac{(1-t) p(\bar{w})}{1-t-s h(v)}<p(\bar{w}) .
$$

Case $4(h(u)=0$ and $h(v)=0)$. We do not use the same $p$ and $\bar{w}$ as in the above cases, but a $q \in S^{*}$ such that $q(u)>0$ and $q(v)>0$, whose existence is guaranteed by the linear independence of $u$ and $v$, and a point $\tilde{w} \in J^{*} h$ such that

$$
q(\tilde{w})=\inf \left\{q(x): x \in J^{*} h\right\} .
$$

We shall prove that $\tilde{w} \in \operatorname{span}(u, v)$. Suppose, on the contrary, that $\tilde{w} \notin$ $\operatorname{span}(u, v)$. Then by Proposition 1 and Corollary $3,0 \in Z^{1}(u, v, 3 \tilde{w})$, but $0 \notin$ 
$\operatorname{co}(u, v, 3 \tilde{w})$. Then, by GK1, there exist $r \geq 0, s \geq 0$, and $t \geq 0$ such that $r+s+t=1$ and

$$
z=r u+s v+t 3 \tilde{w} \in Z^{1}(u, v, 3 \tilde{w}) .
$$

The inequality

$$
\begin{aligned}
5=\|u\|+\|v\|+\|3 \tilde{w}\| & =\|u-z\|+\|v-z\|+\|3 \tilde{w}-z\| \\
& \geq h(z-u)+h(z-v)+h(3 \tilde{w}-z)=3+3 t
\end{aligned}
$$

shows that $t \leq 2 / 3$ and, hence, that either $r>0$ or $s>0$.

Therefore, we have the contradiction

$$
\frac{3 \tilde{w}-z}{\|3 \tilde{w}-z\|} \in J^{*} h
$$

and

$$
q\left(\frac{3 \tilde{w}-z}{\|3 \tilde{w}-z\|}\right)=\frac{(3-3 t) q(\tilde{w})-r q(u)-s q(v)}{3-3 t}<q(\tilde{w})
$$

Proposition 10. Suppose that $X$ satisfies $G K 1$ and that $f, g, h \in S^{*}$ are such that $f+g+h=0$. If $f$ is non-parallel to any segment of $S^{*}$ of length $\geq 1$ and $u \in J^{*} f$, then $g(u)=h(u)=-1 / 2$.

Proof. Let $u^{\prime}, u^{\prime \prime} \in S \cap \operatorname{ker} f$ be linearly independent. Then, for any $\alpha>0, P_{\alpha}=$ $\left\{k \in X^{*}: k\left(u^{\prime}-\alpha u^{\prime \prime}\right)=0\right\}$ is a 2-dimensional subspace of $X^{*}$ that contains $f$, and, by Lemma 6] there is a unique pair $g_{\alpha}, h_{\alpha} \in S^{*} \cap P_{\alpha}$ such that $f+g_{\alpha}+h_{\alpha}=0$.

Since $f$ and $g_{\alpha}$ are linearly independent, $u^{\prime}, u^{\prime \prime} \in \operatorname{ker} f$, and $g_{\alpha}\left(u^{\prime}-\alpha u^{\prime \prime}\right)=0$, we have that $g_{\alpha}\left(u^{\prime \prime}\right) \neq 0$. We may assume that $g_{\alpha}\left(u^{\prime \prime}\right)>0\left(\right.$ and $h_{\alpha}\left(u^{\prime \prime}\right)<0$ ) for every $\alpha>0$. Then it is easy to see that the map $\alpha \in \mathbb{R}_{+} \rightarrow g_{\alpha} \in S^{*}$ is continuous, i.e., it defines a simple arc over $S^{*}$, and we shall see that it is contained in $S^{*} \cap\left\{k \in X^{*}: k(u)=-1 / 2\right\}$.

Let $\left(\alpha_{n}\right) \searrow \alpha$. Since the sequence

$$
\left(p_{\alpha_{n}},-p_{\alpha_{n}}\right)=\left(\frac{g_{\alpha_{n}}-g_{\alpha}}{\left\|g_{\alpha_{n}}-g_{\alpha}\right\|}, \frac{h_{\alpha_{n}}-h_{\alpha}}{\left\|h_{\alpha_{n}}-h_{\alpha}\right\|}\right)
$$

is in the compact set $S^{*} \times S^{*}$, it has a subsequence that converges to $\left(p_{\alpha},-p_{\alpha}\right) \in$ $S^{*} \times S^{*}$.

By the continuity of the maps $\alpha \rightarrow g_{\alpha}$ and $\alpha \rightarrow h_{\alpha}$, the straight lines $\left\{g_{\alpha}+\lambda p_{\alpha}\right.$ : $\lambda \in \mathbb{R}\}$ and $\left\{h_{\alpha}+\lambda p_{\alpha}: \lambda \in \mathbb{R}\right\}$ support $S^{*}$ at $g_{\alpha}$ and $h_{\alpha}$, respectively. Thus, there exist $v_{\alpha} \in J^{*} g_{\alpha}$ and $w_{\alpha} \in J^{*} h_{\alpha}$ such that $p_{\alpha}\left(v_{\alpha}\right)=p_{\alpha}\left(w_{\alpha}\right)=0$. Moreover, for any other $v_{\alpha}^{\prime} \in J^{*} g_{\alpha}$ and $w_{\alpha}^{\prime} \in J^{*} h_{\alpha}$ we have that $\left(g_{\alpha_{n}}-g_{\alpha}\right)\left(v_{\alpha}^{\prime}\right) \leq 0,\left(h_{\alpha_{n}}-h_{\alpha}\right)\left(w_{\alpha}^{\prime}\right) \leq$ 0 . Hence $p_{\alpha}\left(v_{\alpha}^{\prime}\right) \leq 0$ and $p_{\alpha}\left(w_{\alpha}^{\prime}\right) \geq 0$.

We shall prove that $\operatorname{dim}\left[\operatorname{span}\left(u, v_{\alpha}, w_{\alpha}\right)\right]=2$, which, taking into account that $v_{\alpha}$ and $w_{\alpha}$ are linearly independent (Lemma $[$ ) $)$, is equivalent to proving that $p_{\alpha}(u)=0$.

Suppose that $p_{\alpha}(u) \neq 0$, i.e., that $\operatorname{dim}\left[\operatorname{span}\left(u, v_{\alpha}, w_{\alpha}\right)\right]=3$. Then, by Proposition 9 there exist $v_{\alpha}^{\prime} \in J^{*} g_{\alpha}$ and $w_{\alpha}^{\prime} \in J^{*} h_{\alpha}$ such that $\operatorname{dim}\left[\operatorname{span}\left(u, v_{\alpha}^{\prime}, w_{\alpha}\right)\right]=$ $\operatorname{dim}\left[\operatorname{span}\left(u, v_{\alpha}, w_{\alpha}^{\prime}\right)\right]=2$.

By Lemma 7, there exist nonnegative real numbers $r, s, t, r^{\prime}, s^{\prime}, t^{\prime}$ such that $r+$ $s+t=r^{\prime}+s^{\prime}+t^{\prime}=1$ and $r u+s v_{\alpha}^{\prime}+t w_{\alpha}=r^{\prime} u+s^{\prime} v_{\alpha}+t^{\prime} w_{\alpha}^{\prime}=0$. Since both $v_{\alpha}^{\prime}, w_{\alpha}$ and $v_{\alpha}, w_{\alpha}^{\prime}$ are linearly independent pairs, $r$ and $r^{\prime}$ must be positive, and 
we have that $r p_{\alpha}(u)+s p_{\alpha}\left(v_{\alpha}^{\prime}\right)=r^{\prime} p_{\alpha}(u)+t^{\prime} p_{\alpha}\left(w_{\alpha}^{\prime}\right)=0$, which contradicts the aforementioned fact that $p_{\alpha}\left(v_{\alpha}^{\prime}\right) \leq 0$ and $p_{\alpha}\left(w_{\alpha}^{\prime}\right) \geq 0$.

Therefore, every convergent subsequence of $\left(p_{\alpha_{n}}\right)$ converges to the unique $p_{\alpha} \in$ $S^{*}$ such that $p_{\alpha}(u)=0, p_{\alpha}\left(v_{\alpha}\right)=0$ for some $v_{\alpha} \in J^{*} g_{\alpha}$. Moreover, $p_{\alpha}\left(v_{\alpha}^{\prime}\right) \leq 0$ for every $v_{\alpha}^{\prime} \in J^{*} g_{\alpha}$, and hence,

$$
\lim _{\beta \backslash \alpha} \frac{g_{\beta}-g_{\alpha}}{\left\|g_{\beta}-g_{\alpha}\right\|}=p_{\alpha}
$$

Thus,

$$
\begin{gathered}
\lim _{\beta \searrow \alpha} \frac{g_{\beta}(u)-g_{\alpha}(u)}{\left\|g_{\beta}-g_{\alpha}\right\|}=p_{\alpha}(u)=0, \\
\lim _{\beta \searrow \alpha} \frac{g_{\beta}\left(u^{\prime}-\alpha u^{\prime \prime}\right)-g_{\alpha}\left(u^{\prime}-\alpha u^{\prime \prime}\right)}{\left\|g_{\beta}-g_{\alpha}\right\|}=\lim _{\beta \searrow \alpha} \frac{(\beta-\alpha) g_{\beta}\left(u^{\prime \prime}\right)}{\left\|g_{\beta}-g_{\alpha}\right\|} \\
=g_{\alpha}\left(u^{\prime \prime}\right) \lim _{\beta \searrow \alpha} \frac{\beta-\alpha}{\left\|g_{\beta}-g_{\alpha}\right\|}=p_{\alpha}\left(u^{\prime}-\alpha u^{\prime \prime}\right) .
\end{gathered}
$$

Since $g_{\alpha}\left(u^{\prime \prime}\right) \neq 0$, it suffices to see that $p_{\alpha}\left(u^{\prime}-\alpha u^{\prime \prime}\right) \neq 0$ to obtain that

$$
\lim _{\beta \searrow \alpha} \frac{g_{\beta}(u)-g_{\alpha}(u)}{\beta-\alpha}=p_{\alpha}(u)=0,
$$

and, by the mean value theorem, that the continuous function $\alpha \in \mathbb{R}_{+} \rightarrow g_{\alpha}(u)$ is constant.

Suppose, on the contrary, that $p_{\alpha}\left(u^{\prime}-\alpha u^{\prime \prime}\right)=0$. Since $v_{\alpha}$ and $w_{\alpha}$ are linearly independent and $p_{\alpha}\left(v_{\alpha}\right)=p_{\alpha}\left(w_{\alpha}\right)=0$, there exist nonzero real numbers $\lambda$ and $\mu$ such that $\lambda v_{\alpha}+\mu w_{\alpha}=u^{\prime}-\alpha u^{\prime \prime}$, from which it follows that

$$
\begin{aligned}
\lambda g_{\alpha}\left(v_{\alpha}\right)+\mu g_{\alpha}\left(w_{\alpha}\right) & =g_{\alpha}\left(u^{\prime}-\alpha u^{\prime \prime}\right)=0, \\
\lambda h_{\alpha}\left(v_{\alpha}\right)+\mu h_{\alpha}\left(w_{\alpha}\right) & =h_{\alpha}\left(u^{\prime}-\alpha u^{\prime \prime}\right)=0 .
\end{aligned}
$$

Thus $g_{\alpha}\left(w_{\alpha}\right) h_{\alpha}\left(v_{\alpha}\right)=1$ and $\left(g_{\alpha}-h_{\alpha}\right)\left(v_{\alpha}\right)=2=\left\|g_{\alpha}-h_{\alpha}\right\|$. So the segment $\left[g_{\alpha},-h_{\alpha}\right]$ is parallel to $f$, of length 1 , and contained in $S^{*}$, thereby contradicting the hypothesis.

Since we can do the same for $\alpha<0$ and for $u^{\prime \prime}-\alpha u^{\prime}$ instead of $u^{\prime}-\alpha u^{\prime \prime}$, we have obtained that for every $g, h \in S^{*}$ such that $f+g+h=0, g(u)$ is constant. Obviously the constant must be $-1 / 2$, as we wished to show.

Since all the possible points $g$ cannot lie on two planes simultaneously, we have the following immediate consequence.

Corollary 11. If $X$ satisfies $G K 1$ and if $f \in S^{*}$ is non-parallel to a segment of $S^{*}$ of length $\geq 1$, then $J^{*} f$ has a unique element.

Proposition 12. If $X$ satisfies GK1, then $X^{*}$ is rotund (i.e., $X$ is smooth).

Proof. Assume by contradiction that there is a non-void open segment $I \subset S^{*}$, and denote by $P$ the 2-dimensional subspace of $X^{*}$ that contains $I$.

Simple 2-dimensional arguments show that there is at most one segment of $S^{*} \cap P$, of length $>1$, that is parallel to some point of $I$, and that if $f$ and $f^{\prime}$ are two different points of $I$ and $g, h, g^{\prime}, h^{\prime} \in S^{*} \cap P$ are such that $f+g+h=f^{\prime}+g^{\prime}+h^{\prime}=0$, then $g-h$ is non-proportional to $g^{\prime}-h^{\prime}$.

Therefore, we can take $f \in I$ for which the pair $g, h \in S^{*} \cap P$ satisfying $f+g+h=$ 0 is unique and $g-h$ is non-parallel to $I$. Furthermore, since $I$ is an open segment, 
the condition that $g-h$ is non-parallel to $I$ is equivalent to the existence of $c \neq 0$ such that $(g-h)(u)=c$, for every $u \in J^{*} f$.

Since the set of points of $S^{*}$ that are non-parallel to any segment contained in $S^{*}$ is dense in $S^{*}$ [17], there is a sequence $\left(f_{n}\right)$ that converges to $f$ and is formed by points of $S^{*}$ that are non-parallel to any segment of $S^{*}$. By Corollary 11, each $J^{*} f_{n}$ has a unique element $u_{n}$.

Let $P_{n}=\operatorname{span}\left(f_{n}, g\right)$, and let $g_{n}, h_{n}$ be the unique pair of $S^{*} \cap P_{n}$ such that $f+g_{n}+h_{n}=0$. If we suppose that $\left\|g_{n}-g\right\| \leq\left\|h_{n}-g\right\|$, standard continuity arguments show that the sequences $\left(g_{n}\right)$ and $\left(h_{n}\right)$ converge to $g$ and $h$, respectively, and that $\left(u_{n}\right)$ has a subsequence that converges to some $u \in J^{*} f$. Hence, there exists $n \in \mathbb{N}$ such that $f_{n}$ is non-parallel to any segment of $S^{*}$, but $\left|\left(g_{n}-h_{n}\right)\left(u_{n}\right)\right|>$ $\frac{|c|}{2}$, in contradiction with Proposition 10 ,

Corollary 13. If $X$ satisfies $G K 1$, then $X^{*}$ is smooth (i.e., $X$ is rotund).

Proof. Take into account that $X^{*}$ is rotund, and apply Corollary 11

Corollary 14. Assume that $X$ satisfies GK1.

(i) If $f, g, h \in S^{*}$ are such that $f+g+h=0$ and $u=J^{*} f, v=J^{*} g, w=J^{*} h$, then $u+v+w=0$.

(ii) If $u, v, w \in S$ are such that $u+v+w=0$ and $f=J u, g=J v, h=J w$, then $f+g+h=0$.

Proof. (i) This follows from $f+g+h=0, f(u)=g(v)=h(w)=1$, and $f(v)=$ $f(w)=g(u)=g(w)=h(u)=h(v)=-\frac{1}{2}$.

(ii) A simple continuity argument shows that there exists $g^{\prime} \in S^{*}$ such that $\left\|f+g^{\prime}\right\|=1$ and $v^{\prime}=J g^{\prime}$ belongs to $\operatorname{span}(u, v)$. Let $h^{\prime}=-f-g^{\prime}$ and $w^{\prime}=J h^{\prime}$. It follows from (i) that $u+v^{\prime}+w^{\prime}=0$, which, together with $u+v+w=0$, implies that $v=v^{\prime}, w=w^{\prime}$. Hence, $g=g^{\prime}, h=h^{\prime}$.

As in Proposition 1, certain arguments concerning areas which we shall use in the next, final theorem are connected with results of Fasbender [10 for the classical Fermat problem (see, e.g., [14, p. 58]).

Theorem 15. A real normed space $X$ of dimension $\geq 3$ is an inner product space if and only if

$$
Z^{1}(u, v, w) \cap \operatorname{co}(u, v, w) \neq \emptyset, \quad \text { for every } u, v, w \in X .
$$

Proof. On the one hand, it is well known and easy to see that an inner product space satisfies GK1.

On the other hand, we have previously proved that if $X$ satisfies GK1, then it is regular (smooth and rotund) and such that

$$
u, v, w \in S, \quad u+v+w=0, \quad J u=f, \quad J v=g, \quad J w=h
$$

imply

$$
f(v)=f(w)=g(u)=g(w)=h(u)=h(v)=-\frac{1}{2} .
$$

Making use of this, we shall see that every two-dimensional subspace $Y$ of $X$ is an inner product space, i.e. (identifying $Y$ with $\mathbb{R}^{2}$ ), that $S \cap Y$ is an ellipse.

Denote by $\prec$ the positive orientation of the plane $Y$. For $u_{0} \in S \cap Y$, let $u_{0} \prec u_{\theta}$ be the point of $S \cap Y$ that forms an angle $0 \leq \theta \leq \pi$ with $u_{0}$ and let $v_{\theta}, w_{\theta}$ be the unique points of $S \cap Y$ that satisfy $w_{\theta} \prec u_{\theta} \prec v_{\theta}$ and $u_{\theta}+v_{\theta}+w_{\theta}=0$. 
Since $S \cap Y$ is a convex regular curve, $u: \theta \in[0, \pi] \rightarrow u_{\theta}$ is continuously differentiable and it is easy to see that $v: \theta \in[0, \pi] \rightarrow v_{\theta}$ and $w: \theta \in[0, \pi] \rightarrow w_{\theta}$ are continuous and of bounded variation (note that $S \cap Y$ is a convex curve and $v$, $w$ are increasing, i.e., such that $0 \leq \theta_{1}<\theta_{2} \leq \pi$ implies $v_{\theta_{1}} \prec v_{\theta_{2}}$ and $w_{\theta_{1}} \prec w_{\theta_{2}}$ ). Hence, all the Riemann-Stieltjes integrals that will appear are meaningful.

As usual, for a pair $x=\left(x_{1}, x_{2}\right)$ and $y=\left(y_{1}, y_{2}\right)$ of points of $Y$ such that $x \prec y$, we denote by $x \wedge y=x_{1} y_{2}-x_{2} y_{1}$ the area of the parallelogram with sides $x$ and $y$. Then $y \wedge x=-x \wedge y, x \wedge(y+z)=x \wedge y+x \wedge z$, etc.

Note that $u_{\theta}+v_{\theta}+w_{\theta}=0$ implies that $u_{\theta} \wedge\left(u_{\theta}+v_{\theta}+w_{\theta}\right)=v_{\theta} \wedge\left(u_{\theta}+v_{\theta}+w_{\theta}\right)=0$ and, hence,

$$
u_{\theta} \wedge v_{\theta}=v_{\theta} \wedge w_{\theta}=w_{\theta} \wedge u_{\theta} .
$$

Write $J u_{\theta}=f_{\theta}$. It follows from $f_{\theta}\left(v_{\theta}-w_{\theta}\right)=0$ that, for every partition $0=\alpha_{0}<\alpha_{1}<\ldots<\alpha_{n}=\alpha$ of the interval $[0, \alpha]$, with $0<\alpha \leq \pi$, there exist $\alpha_{0}<\beta_{1}<\alpha_{1}<\beta_{2}<\alpha_{2}<\ldots<\alpha_{n-1}<\beta_{n}<\alpha_{n}$ such that

$$
f_{\beta_{k}}\left(v_{\beta_{k}}-w_{\beta_{k}}\right)=f_{\beta_{k}}\left(u_{\alpha_{k}}-u_{\alpha_{k-1}}\right)=0 \quad(k=1, \ldots, n) .
$$

Then,

$$
\left(v_{\beta_{k}}-w_{\beta_{k}}\right) \wedge\left(u_{\alpha_{k}}-u_{\alpha_{k-1}}\right)=0 \quad(k=1, \ldots, n)
$$

and, hence,

$$
\int_{0}^{\alpha}(v-w) \wedge d u=0
$$

which, taking into account that $u_{\theta}+v_{\theta}+w_{\theta}=0$, can be written

$$
\int_{0}^{\alpha}(u+2 v) \wedge d u=0
$$

Analogously,

$$
\int_{0}^{\alpha}(w-u) \wedge d v=\int_{0}^{\alpha}(-2 u-v) \wedge d v=\int_{0}^{\alpha}(u-v) \wedge d w=0,
$$

and, therefore,

$$
\begin{gathered}
\int_{0}^{\alpha}(v-w) \wedge d u+\int_{0}^{\alpha}(w-u) \wedge d v+\int_{0}^{\alpha}(u-v) \wedge d w \\
=\int_{0}^{\alpha} d[(v-u) \wedge(v-w)]=\left(v_{\alpha}-u_{\alpha}\right) \wedge\left(v_{\alpha}-w_{\alpha}\right)-\left(v_{0}-u_{0}\right) \wedge\left(v_{0}-w_{0}\right)=0,
\end{gathered}
$$

i.e., the function

$$
\theta \in[0, \pi] \rightarrow\left(u_{\theta}-v_{\theta}\right) \wedge\left(v_{\theta}-w_{\theta}\right)=u_{\theta} \wedge v_{\theta}+v_{\theta} \wedge w_{\theta}+w_{\theta} \wedge u_{\theta}
$$

is constant and, since $u_{\theta} \wedge v_{\theta}=v_{\theta} \wedge w_{\theta}=w_{\theta} \wedge u_{\theta}$, the functions

$$
\theta \rightarrow u_{\theta} \wedge v_{\theta}, \quad \theta \rightarrow v_{\theta} \wedge w_{\theta}, \quad \theta \rightarrow w_{\theta} \wedge u_{\theta}
$$

are also constant.

Therefore,

$$
\int_{0}^{\alpha} d(u \wedge v)=\int_{0}^{\alpha} u \wedge d v-\int_{0}^{\alpha} v \wedge d u=0
$$


and, hence,

$$
\int_{0}^{\alpha} u \wedge d u=-2 \int_{0}^{\alpha} v \wedge d u=-2 \int_{0}^{\alpha} u \wedge d v=\int_{0}^{\alpha} v \wedge d v .
$$

Furthermore, since $\theta \in[0, \pi] \rightarrow u_{\theta}$ is continuously differentiable, $\theta \in[0, \pi] \rightarrow$ $u_{\theta} \wedge v_{\theta}$ is constant, and $\theta \in[0, \pi] \rightarrow v_{\theta}$ is continuous and increasing, this last function is also continuously differentiable.

Then, the above Riemann-Stieltjes integrals can be reduced to ordinary Riemann integrals, and it follows from

$$
\begin{gathered}
\int_{0}^{\alpha}(u+2 v) \wedge d u=\int_{0}^{\alpha}(u+2 v) \wedge u^{\prime}=0 \\
\int_{0}^{\alpha}(-2 u-v) \wedge d v=\int_{0}^{\alpha}(-2 u-v) \wedge v^{\prime}=0
\end{gathered}
$$

that there exist positive continuous functions $p:[0, \pi] \rightarrow \mathbb{R}$ and $q:[0, \pi] \rightarrow \mathbb{R}$ such that

$$
u^{\prime}=p(u+2 v), \quad v^{\prime}=q(-2 u-v) .
$$

Hence

$$
\begin{aligned}
& \int_{0}^{\alpha} u \wedge d u=\int_{0}^{\alpha} u \wedge u^{\prime}=\int_{0}^{\alpha} u \wedge p(u+2 v) \\
= & \int_{0}^{\alpha} v \wedge d v=\int_{0}^{\alpha} v \wedge v^{\prime}=\int_{0}^{\alpha} v \wedge q(-2 u-v),
\end{aligned}
$$

and, since $u \wedge v$ is constant, $p=q$.

Finally, solving the simple system of differential equations

$$
\begin{gathered}
u_{1}^{\prime}=p\left(u_{1}+2 v_{1}\right), \\
u_{2}^{\prime}=p\left(u_{2}+2 v_{2}\right), \\
v_{1}^{\prime}=-p\left(2 u_{1}+v_{1}\right), \\
v_{2}^{\prime}=-p\left(2 u_{2}+v_{2}\right),
\end{gathered}
$$

with the non-restrictive initial conditions $u_{0}=(1,0), v_{0}=\left(-\frac{1}{2}, \frac{\sqrt{3}}{2}\right)$, we obtain

$$
u_{1}^{2}+v_{1}^{2}+u_{1} v_{1}=u_{2}^{2}+v_{2}^{2}+u_{2} v_{2}=\frac{3}{4}
$$

and this, together with

$$
u_{1} v_{2}-u_{2} v_{1}=\frac{\sqrt{3}}{2}
$$

gives

$$
S \cap Y=\left\{\left(u_{1}, u_{2}\right): u_{1}^{2}+u_{2}^{2}=1\right\}
$$

as we wished to show.

Remark 16. It is worth mentioning that much of the work in this paper (cases 2, 3, 4 of Proposition 9. many details in Proposition 11 Proposition [12 ... ) is due to the, a priori, non-regularity of $X$. Our task would have been considerably easier with the additional hypothesis that $X$ is rotund and smooth. 


\section{ACKNOWLEDGEMENT}

The authors thank their colleague D. Yáñez for an interesting hint relative to the last part of the final theorem, and the referee for several remarks that improved the paper.

\section{REFERENCES}

1. D. AmIR, "Characterization of Inner Product Spaces", Birkhäuser-Verlag, Basel, 1986. MR 88m:46001

2. M. Baronti, E. Casini and P. L. Papini, Equilateral sets and their central points, Rend. Mat. Appl. (7) 13 no. 1, (1993), 133-148. MR 94m:46020

3. C. Benítez, M. Fernández, And M. L. Soriano, Location of the 2-centers of three points, Rev. R. Acad. Cienc. Exact. Fis. Nat.(Esp) 4 (2000), no. 4, 515-517.

4. C. Benítez, M. Fernández, and M. L. Soriano, Weighted $p$-centers and the convex hull property, Numer. Funct. Anal. and Optimiz. 23 (2002), nos. 1 and 2, 39-45.

5. C. Benítez And D. YÁÑEz, A Two-dimensional characterization of inner product spaces. Preprint.

6. V. Boltianski, H. Martini, and V. Soltan, "Geometric Methods and Optimization Problems", Kluwer Academic Publishers, Dordrecht, 1999. MR 2000c:90002

7. D. Cieslik, "Steiner Minimal Trees", Kluwer Academic Publishers, Dordrecht, 1998. MR 99i:05062

8. R. Durier, The Fermat-Weber problem and inner product spaces, J. Approx. Theory 78 (1994), no. 2, 161-173. MR 95g:41048

9. R. Durier, Convex hull properties in location theory, Numer. Funct. Anal. and Optimiz. 15 (5 and 6), (1994), 567-582. MR 95g:46038

10. E. Fasbender, Über die gleichseitigen Dreiecke, welche um ein gegebenes Dreieck gelegt werden können, J. reine angew. Math. 30 (1846), 230-231.

11. P. Fermat, "Oeuvres", Ed. M. M. P. Tannery, Ch. Henry, Tome I, Paris, Gauthier-Villars et Fils, 1891.

12. A.L. Garkavi, On the Chebyshev center and the convex hull of a set, Uspekhi Mat. Nauk 19 (1964), No. 6, 139-145. (Russian) MR 30:5221

13. V. KLeE, Circumspheres and inner products, Math Scand. 8 (1960), 363-370. MR 23:A2734

14. H. W. Kunn, ' "Steiner's" problem revisited', in G. B. Dantzig and B. C. Eaves (eds.), Studies in Optimization, MAA Studies in Mathematics, Vol. 10, Mathematical Assoc. of America, 1974, 52-70. MR 57:18835

15. H. W. Kunn, Nonlinear programming: a historical view, SIAM-AMS Proceedings, Vol. 9 (1976), 1-26. MR 53:7485

16. G. Lewicki, On a new proof of Durier's theorem, Quaestiones Math. 18 (1995), 287-294. MR 96f: 46033

17. T. J. McMinn, On the line segments of a convex surface in $E_{3}$, Pacific J. Math. 10 (1960), 943-946. MR 22:4987

18. I. Singer, "Best Approximation in Normed Linear Spaces by Elements of Linear Subspaces", Springer-Verlag, New York, 1970. MR 42:4937

19. L. VESELÝ, A characterization of reflexivity in the terms of the existence of generalized centers, Extrac. Math. 8 No. 2-3, (1993), 125-131. MR 95i:46024

20. R. E. Wendell and A. P. Hurter, Location theory, dominance, and convexity, Oper. Res. 21 (1973), 314-321. MR 50:3897

Departamento de Matemáticas, Universidad de Extremadura, 06071 Badajoz, Spain

E-mail address: cabero@unex.es

Departamento de Matemáticas, Universidad de Extremadura, 06071 Badajoz, Spain

E-mail address: ghierro@unex.es

Departamento de Matemáticas, Universidad de Extremadura, 06071 Badajoz, Spain

E-mail address: 1soriano@unex.es 